\title{
De utilitate et necessitate geographiae: Umweltforschung - mit oder ohne Geographie?
}

\author{
Dieter Brunnschweiler
}

Vor dreihundert Jahren hat Varenius (1672) unter den auszeichnenden Merkmalen der Geographie deren Nützlichkeit und Notwendigkeit (utilitas et necessitas) hervorgehoben, und zwar weil die Erkenntnisse der Geographie weder von den Naturnoch von den Geisteswissenschaften entbehrt werden könnten, falls diese nicht eine Verzögerung im Fortschritt ihrer eigenen Studien in Kauf nehmen wollten (si absque remora progredi voluerint in suis studiis: op. cit., Caput I, Prolegomena). Dafür, daß dieser Vorgänger der wissenschaftlichen Geographie von seiner Zeit ernst genommen wurde, zeugt der Name des Herausgebers der englischen Ausgabe von Varenius' "Geographia Generalis» - Isaac Newton.

Es dürfte im Rahmen des vorliegenden Symposiums über die Prinzipien der Geographie nicht nur von methodologischem Interesse sein, sondern auch ein passender Zeitpunkt, einige Betrachtungen über die von Varenius postulierte Gemeinnützigkeit unseres Faches anzustellen. Denn wir leben ja in einer Periode, in welcher man sich in aller Herren Länder als Folge einer Reihe von Ereignissen, die alle das Verhältnis zwischen Mensch und Erde, vom kleinsten, weltweiten bis zum größten, persönlichen Maßstab betreffen, mit Bestürzung bewußt wird, $\mathrm{da} \beta$ die so oft besungene "schöne, weite Welt» im Begriffe ist, beide schmückenden Beiwörter bald zu verlieren - wovor der Geograph auf Planet VI schon lange warnte, als er dem Kleinen Prinzen am Beispiel der ephemeren Rose zu verstehen gab, daß auch schöne Dinge «in Gefahr raschen Verschwindens» seien (Saint-Exupéry, 1943).

\section{Der Ruf nach einer Umweltlehre}

Dieses ganz plötzlich entsprungene Welt- und Umweltbewußtsein von seiten jedermanns mag den Geographen eigenartig anmuten, denn er ist ja von Berufs wegen erdkundig und kann auf eine lange Serie von Abhandlungen verweisen, in welchen das prekäre Gleichgewicht im terrestrischen Habitat von allen möglichen Gesichtspunkten behandelt wird. Aber da die neue Bewegung versucht, sich mittels eines theoretischen und methodologischen Grundgerüstes zu einer eigenen Wissenschaft emporzuarbeiten, die man in ihren ernsten Bestrebungen wohl als Umweltforschung, im Zeterge- schrei ihrer Mitläufer aber besser als Allerweltslehre bezeichnen kann, und in deren Terminologie Begriffe erscheinen, die uns altbekannt, um nicht zu sagen abgedroschen vorkommen - Umweltfaktoren, Landschaftsharmonie, "human ecology, carrying capacity» zum Beispiel - ist die Frage nicht ob, sondern wie die Geographie zu der von den verschiedensten akademischen Sparten aufgenommenen «ökologischen Revolution» Stellung nehmen soll.

Nun haben wir uns sicherlich nie eingebildet, daß die Geographie trotz der universalen Spannweite ihres Untersuchungsfeldes die einzige und allein seligmachende Umweltwissenschaft sei. Ihrem Objekt nach Mensch und Natur betrachtend, in der Methode analytisch und integrierend, im Umfang vierdimensional und als altehrwürdige Wissenschaftsmutter sozusagen auf natürliche Weise für Nachkommen verantwortlich, scheint sie für eine Aufteilung ihres komplexen Sachgehaltes für spezielle Untersuchungen prädestiniert zu sein, ohne indessen jemals Gefahr zu laufen, ihr ursprüngliches Arbeitsfeld, den festen Boden der Erdlandschaft selbst, zu verlieren. So sieht Boesch (1962) die heutige Spezialisierungstendenz aller Wissenschaften als eine Entwicklung, welche die Geographie in verstärktem Maße mitmachen muß.

«...da im geosphärischen Bereich mehr und vielseitigere Dinge zusammenkommen als irgendwo sonst. Die Fülle des vorhandenen Tatsachenmaterials bedrängt den Geographen in einem solchen $\mathrm{Maße}$, daß er je länger je mehr gezwungen ist, seine eigentliche Forschungsarbeit an der Peripherie, das heißt in den einzelnen Teilgebieten, einzuschränken oder gar ganz aufzugeben und sich in erster Linie auf Besonderheiten, welche durch das Zusammentreten der einzelnen Bausteine entstehen, zu konzentrieren... Die Zeiten einer wahrhaft umfassenden Beherrschung aller Sphären sind (aber) auf immer verschwunden.»

Diese nüchternen Feststellungen eines der ausgesprochensten Vertreter positiven Denkens in Sachen Geographie mag vorerst überraschen, doch sind diese erstens nur voll verständlich im Zusammenhang mit der nachfolgenden Gedankenführung, in welcher zur Landschaftsanalyse als Kernthema 
des geographischen Arbeitens aufgebaut wird, und zweitens könnte der abschließende Satz auf alle jene Wissenschaften angewendet werden, die als Folge der sprunghaften Entwicklung in moderner Informationsbeschaffung und Untersuchungstechnik $\mathrm{zu}$ fortschreitender Arbeitsteilung gezwungen sind.

Was nun die Frage anbetrifft, wie weit sich die Geographie bisher mit der Umwelt als sozialproblematischem und ordnungsbedürftigem Forschungsobjekt beschäftigt hat, wäre in erster Linie auf die praktische Auswertung vieler in der Geographie gewonnener Erkenntnisse in der Planung zu verweisen, wobei es irrelevant ist, ob der Geograph selbst an letzterer beteiligt ist. Von zunehmender Wichtigkeit ist die angewandte Geographie, zweifelsohne eine völlig genügende und klare Bezeichnung des strategischen Armes unserer Wissenschaft (siehe hierzu die methodologisch korrekte Ausklammerung der «applizierten» von der «reinen» Geographie bei Winkler, 1970, p. 13). Daß der Geograph aber vor allem Diagnostiker ist und bleiben muß, hat wiederum Boesch betont, in dessen folgenden Ausführungen die Idee über das gesunde "Funktionieren einer Landschaft» - Inbegriff seiner Zürcher Schule - alle nötigen Elemente für eine geographisch konzipierte Ökologie enthält.

«... Eingriffe in die formale Struktur (führen aber) dazu, daß ein unter Umständen seit langem eingespieltes Gleichgewicht in tragischer Weise gestört wird und sich als Folge einer Kettenreaktion unvorhergesehene Störungen ergeben... Der Geograph steht allen diesen Veränderungen wie der Arzt dem kranken Körper gegenüber; was hier in gärendem Wandel begriffen ist und in irgend einer Weise wieder in Einklang gebracht werden sollte, war seit jeher sein Untersuchungsund Forschungsobjekt, das er sowohl in seinen Teilen wie auch als Ganzes zu verstehen versucht» (op. cit., p. 293).

Einer der besten in der Fachliteratur niedergelegten Beweise, daß sowohl Natur- wie Kulturgeographen mit hoher Autorität an die Lösung von Umweltproblemen beitragen können, ist erbracht in der Qualität und Quantität der geographischen Beiträge zu dem denkwürdigen, nun schon eine halbe Generationsspanne zurückliegenden Symposium an der Princeton University über "die Rolle des Menschen in der Veränderung des Erdantlitzes» («Man's Role in Changing the Face of the Earth»; Thomas, 1956). Für ein Exposé des in diesem Band enthaltenen reichen Gedankenschatzes reicht der Raum hier nicht aus, doch sei der damaligen Besorgnis der Geographen um die Zukunft der Ökumene mit der humanistischen Aufforderung Altmeister Sauers Ausdruck gegeben:
"Was wir brauchen ist eine Ethik und Ästhetik, aufgrund derer der Mensch, mit Klugheit und Mäßigung, der Nachwelt eine gute Erde hinterlassen kann» (op. cit., p. 68: in wörtlicher Übersetzung aus dem Englischen).

\section{Amerikanische Landschaftsproblematik}

Es ist nicht von ungefähr, daß der Kreuzzug zur Rettung der Umwelt in den Vereinigten Staaten von Amerika sein Hauptquartier aufgeschlagen hat. Die herrliche Naturlandschaft dieses Kontinentes ist vielerorts im Zuge der schonungslosen Kolonisation in solchem Grade verstümmelt worden, daß das geographische Resultat sozusagen als «Unkulturlandschaft" angesprochen werden könnte. Niemand hat die Rücksichtslosigkeit des Amerikaners seiner Wohnstattumgebung gegenüber - in krassem Gegensatz zu seiner Erholungslandschaft, die er sich in den prächtigen Nationalpärken erhalten hat - besser erkannt und beschrieben als Boesch (1955), wenn er in souveräner Methodik, wie in allen seinen Studien zur amerikanischen Landschaftsgeschichte - die leider hier viel zu wenig bekannt sind - die heutige Krise klar auf die wesentlichen entwicklungsgeschichtlichen Ursachen zurückführt und den amerikanischen Geographen vorwirft, daß sie vor lauter Gegenwart die Vergangenheit nicht sähen (op. cit., p. 13); und andernorts (Boesch, 1956), in seinem an die allgemeine Leserschaft gerichteten Amerikawerk, übt er in an Deutlichkeit nichts zu wünschen übrig lassender Sprache an der mangelnden Landschaftsästhetik des Durchschnittsamerikaners Kritik.

Wenn sich auch die amerikanische Nation dieser Situation längst bewußt war - es sind über hundert Jahre her, seit die ersten schwarzen Wasser den Susquehanna hinuntergeflossen sind -, und trotz einer langen Tradition von Landschaftsschutz, Regional- und Stadtplanung, brauchte es regelrechte "Kulturlandschaftskatastrophen» wie vergiftende Inversionsnebel, Seenpollution und Aufstände in den «von Mehltau befallenen Quartieren» («Boesch, op. cit., p. 236), den «blighted districts» der Großstädte, um sie aus der Lethargie herauszureißen.

\section{Der Neo-«Environmentalism» in den Staaten}

Diese Zeilen sind geschrieben unter dem Einfluß eines nun vielleicht fünf Jahre alten Wort- und Bildschwalles, durch welchen man in Amerika über die Notwendigkeit der «Restauration der Qualität unserer Umwelt» ( «Restoring the Quality of Our Environment») orientiert wird. Der Titel des ersten Berichtes der Wissenschaftskommission des Präsidenten betreffend «Umweltfragen» (President's Scien- 
ce Advisory Committee, 1965) deutet die hohe Ebene an, auf welcher die politische Kampagne geführt wird1. "Environmental protection" (Schutz der natürlichen Umwelt), "ecological revolution, pollution control» (wobei jegliche Art von Landschaftsverunschönerung gemeint ist), «zero-population growth" sind einige der Schlagwörter, Leute aus allen Ständen und Berufen deren Verfechter. Allen voran marschieren die tat- und schlagbereiten Aktivisten des Naturschutzkorps, welche wahre Feldzüge im Namen der «ökotaktischen Wissenschaft» durchführen, «im Kampfe gegen die Feinde der Erde» (Mitchell, 1970).

Man mag hier von einem "environmentalism in extremis» sprechen, bei welchem das deterministische Element darin besteht, daß die Menschheit im Begriffe ist, infolge Miß- und Übernutzung der Naturschätze eine Todeslandschaft - ein Nekrochor zu errichten und daß sie den Jüngsten Tag selbst vorbereitet, nicht bösen Willens, aber uneingedenk des relativ einfachen Prozesses der natürlichen Vermehrung. Mehr zu sagen wäre «Sprachpollution».

\section{Die Stellung der amerikanischen Geographie zur Umweltfrage}

Vorläufig scheint es die geographische Fachschaft in den Staaten, von einigen Ausnahmen abgesehen', nicht eilig $\mathrm{zu}$ haben, sich dem «ecology movement" anzuschließen, obschon Forschungsprojekte, die sich in irgend einer Weise mit «environmental quality" befassen, auf dem wissenschaftlichen Finanzmarkt eine Hausse genießen. Vereinzelte Vertreter des Fachkreises haben allerdings öffentlich zum "environmental issue» Stellung genommen, bei dem es sich natürlich nicht um die durch die ganze Geschichte der Geographie gehende und keineswegs verstummte Frage über "environmental influence» handelt (siehe $\mathrm{z}$. B. die neuliche Uberprüfung der deterministischen Milieulehre von Lewthwaite, 1965). Hare (1970) hob in aller Sachlichkeit und Ruhe in der einflußreichen Zeitschrift "Science» die Verflochtenheit des Umweltproblems hervor, zu dessen Lösung weder die Ökologie, noch die Soziologie, die Geographie oder irgend eine andere Disziplin allein viel beitragen könne, alle vereint aber, unter Hintanstellung der fachlichen Idiosynkrasien, eine starke wissenschaftliche Front gegen die asoziale Ausbeutung der Umwelt bilden würden. Wie Hare verzichtet auch Burton (1968) darauf, der Profession irgendwelche Vorschläge $\mathrm{zu}$ machen, wie sie sich gegenüber dem neo-environmentalistischen Zeitgeist verhalten soll. Vielmehr läßt er unter Zitierung verschiedenster umweltkritischer, auf Lösung akuter Regionalprobleme bedachter Arbeiten geo- graphischer Autoren durchblicken, daß sie im löblichen Werke fortfahren solle wie bisher.

Wohl die bekannteste Interessengruppe innerhalb der Geographie, die sich methodologisch mit dem Umweltkonzept beschäftigt, ist jene, deren Vertreter sich «environmental perceptionists» nennen. Saarinen (1969) sieht die wesentliche Aufgabe einer zukünftigen Umweltempfindungslehre - «the science of environmental perception" - entweder in

«1. dem Bestimmen der maßgebenden Faktoren in realen, die Umwelt betreffenden Entscheidungen des Menschen, wofür eine Theorie erst noch entwickelt werden muß» oder in

«2. dem genauen Messen von beschränkten und isolierten Aspekten in künstlichen «Umwelten», mit dem Ziel, hernach Schritt für Schritt eine rigorose Methodologie und Theorie aufzubauen, welche dann auf die reale Welt anwendbar sind" (op. cit., p. 3; in wörtlicher Úbersetzung aus dem Englischen).

Diese ins Gebiet der Verhaltenswissenschaft gehörenden Fragestellungen mögen vorerst allzu esoterisch erscheinen, doch sie gehen von der utilitaristischen Überlegung aus, daß ein vom Geographen erarbeitetes Informationssystem über das Verhalten der Bevölkerung gegenüber den existierenden Umweltproblemen im lokalen, regionalen und nationalen Rahmen Wesentliches zu deren allmählicher Lösung beitragen kann. Verschiedene Pionierarbeiten in dieser Richtung, unter anderen die Studien über das naturbedingte Risiko "natural hazard» in wiederholt von Naturkatastrophen heimgesuchten Gebieten (z. B. White, 1966), haben weit über die Fachgrenzen hinaus Beachtung gefunden ${ }^{3}$.

Auch Loewenthals (1968) epistemologische Untersuchungen über den "Landschaftsgeschmack» ("landscapes are formed by landscape tastes», op. cit., p. 6) gehören in die Kategorie der Wahrnehmungsstudien. Hier wird versucht, das Kollektivempfinden der Bevölkerung gegenüber der sichtbaren Umwelt festzustellen, mit dem Zweck, dieses in eine wirkungsvolle Landschaftspolitik einzubauen:

"Um durchschlagskräftig zu sein, müssen Planung und Baugestaltung (,design') auf das tiefste damit vertraut sein, wie die Leute in bezug auf ihre Umgebung denken und fühlen. Dies setzt eine intime Kenntnis von sozialer und intellektueller Geschichte, von Psychologie und Philosophie wie auch von Kunst und Anthropologie voraus. Alle diese tragen das Ihrige bei zu unserem Verständnis über die Welt, in der wir leben, über die Art und Weise, wie Wahrnehmung und Wertschätzung das Handeln beeinflussen und wie das Handeln die Umwelt ändert» (op. cit., p. 88; wörtlich übersetzt aus dem Englischen). 


\section{Ceterum censeo...}

Beschrieben wurden anfängliche, aber doch ernstliche Bestrebungen, von einer beschaulichen $\mathrm{zu}$ einer pragmatischen, ja sogar nomothetischen Geographie zu gelangen, in welcher die gegenseitigen Rückwirkungen im System Mensch-Umwelt qualitativ wahrnehmbar und quantitativ meßbar wären. Die verspätete Einsicht in öffentlichen wie in akademischen Kreisen, daß das Studium der kausalen Zusammenhänge zwischen menschlichem Handeln und landschaftlicher Ausstattung - das Alpha und Omega geographischer Fragestellung - nicht allein Würde (dignitas), Freude (iucundum quoque), und wahrhaftige Erholung (honesta recreatio) mit sich bringt, um den Gedankenkreis mit Varenius (op. cit.) zu schließen, sondern auch nützlich und im wahrsten Sinne des Wortes lebensnotwendig ist, mag die Geographie unter Umständen aus ihrer zu lange genossenen "splendid isolation" herauslösen und dazu beitragen, ihre Arbeitsfront schärfer als bisher in Richtung praktischer Probleme zu orientieren.

\section{Anmerkungen}

1 Im Zeichen der Zeit wurde auch ein höchster Verwaltungsarm geschaffen, der für nationale Umweltfragen verantwortlich ist: "The Environmental Science Services Administration, ESSA " (Dienstabteilung für Umweltforschung). In der Privatindustrie bilden sich Forschungsgruppen, die sich für ecological oder environmental systems analysis anbieten (ökologische oder umweltorientierte Systemanalyse). An Universitäten können Lehrpläne von Geoscience (Erdkunde?!), Environmental Sciences (Umweltwissenschaften) oder Environmental Engineering (Umweltingenieurkunst) eingehalten werden.

2 So berührt es $\mathbf{z}$. B. sonderbar, wenn ein so verdienter Methodologe wie R. E. Dickinson sein jüngstes Werk, "The Study of Man's Environment», mit dem Haupttitel «Regional Ecology» überschreibt (Wiley \& Sons, New York, 1970).

3 Besonders die unter White von der "Chicago Schule» in Dissertationen und Spezialarbeiten durchgeführten Studien über Flutgefährdung und -verhütung in den Vereinigten Staaten sind hier zu nennen. White ist übrigens kürzlich zum Direktor eines Forschungsinstituts für Umweltfragen an der Universität von Colorado gewählt worden.

\section{Résumé}

L'auteur décrit d'entrée de cause les efforts faits en vue de passer des formes d'une géographie contem- plative à celles d'une géographie pragmatique sinon nomothétique, dans laquelle les efforts réciproques dans le système homme - environnement peuvent être mesurés, tant sur le plan qualitatif que quantitatif. La reconnaissance tardive, tant dans le grand public que dans les milieux académiques, de la corrélation causale entre le comportement humain et la configuration du paysage répond à une nécessité. Puisse-t-elle sortir la géographie de son «splendide isolement» et contribuer à ce que son travail s'oriente vers des problèmes pratiques.

\section{Literatur}

Boesch, H., 1955: Amerikanische Landschaft. Vierteljahresschrift der Naturforschenden Gesellschaft in Zürich. 99, 66 pp.

- 1956: USA. Die Erschließung eines Kontinentes. Kümmerly \& Frey, Bern, 272 pp.

- 1962: Zur Stellung der modernen Geographie. Geographica Helvetica, 17, 4, pp. 288-293.

Burton I., 1968: The Quality of the Environment: A Review. Geographical Review, Vol. 58, No. 3, pp. 472-481.

Hare F. K., 1970: How Should We Treat Environment? Science, Vol. 167, pp. 352-355.

Lewthwaite G. R., 1966: Environmentalism and Determinism: A Search for Clarification. Annals of the Association of American Geographers, 56, 1, pp. 1-23.

Loewenthal D., 1968: The American Scene. Geographical Review, 58, 1, pp. 61-88.

Mitchell J. G., 1970: Ecotactics: The Sierra Club Handbook for Environmental Activists. Pocket Books, New York, 288 pp.

Saarinen T. F., 1969: Perception of Environment. Association of American Geographers, Commission on College Geography, Resource Paper, 5. Washington, $37 \mathrm{pp}$.

Saint-Exupéry, A. de, 1943: Le Petit Prince. Reynal and Hitchcock, New York, 91 pp.

Thomas W. L., 1956: Man's Role in Changing the Face of the Earth. The University of Chicago Press, Chicago, 1193 pp.

President's Science Advisory Committee, 1965: Restoring the Quality of Our Environment. Washington, D. C., $317 \mathrm{pp}$.

Varenius B., 1672: Geographia Generalis. Cambridge, $511 \mathrm{pp}$.

White G., 1966: Optimal Flood Damage Management. In: Water Research, Kneese A. V. und Smith S. S. Eds. Baltimore, pp. 251-269.

Winkler E., 1970: A Possible Classification of the Geosciences. In: Geoforum, 1, 1, pp. 9-17. 